\title{
Study and Realization of SVPWM Algorithm's Key Technology
}

\author{
Liu Bingyou* and Yang Huicheng
}

\begin{abstract}
Key Lab of Electric and Control of Anhui Province, College of Electrical Engineering Anhui Polytechnic University, Wuhu, 241000, P.R. China
\end{abstract}

\begin{abstract}
Control algorithm is the core of AC servo control system. In order to improve the performances of system, on the basis of vector control system and space vector pulse width modulation (SVPWM), inverse matrix and projection of $U_{\text {out }}$ on plane rectangular coordinate system were used to calculate the nonzero vector movement time $t 1, t 2$. Then, a method for calculating vector switch points $T_{c m 1}, T_{c m 2}, T_{c m 3}$ was given. Finally, a judgment method for sectors of the vector was given based on the relationship between index number and sectors. Simulations were carried out and results show that the system exhibits better performances.
\end{abstract}

Keywords: Key Technology, Nonzero Vector, Sector, SVPWM, Vector Movement Time.

\section{INTRODUCTION}

Good dynamic and steady-state performance are very important in industrial fields, for example, high positioning accuracy fields, rapid response speed fields, wide speed range (above 1:10000) fields, high torque output fields, etc. [1,2]. The key technology of realizing the space vector pulse width modulation (SVPWM) algorithm plays an important role in improving the performances. Therefore, the SVPWM algorithm is widely studied by domestic and foreign scholars.

Nowadays most scholars focus on the intelligent control strategy, the adaptive control strategy, the sliding mode variable structure control strategy, the direct torque control strategy and the vector control strategy. Some achievements have been made in the research of these control strategies, and the vector control strategy among them has many advantages, like linear characteristics of torque output, high utilization rate of current and easy realization of regulator [3]. Therefore, the vector control strategy is widely applied in most fields.

In this article, a method for calculating sectors of the vector is proposed and a judging method for the vector switch points $T_{c m 1}, T_{c m 2}, T_{c m 3}$ is given. The nonzero vector movement time $t 1, t 2$ is calculated. And a method for calculating sectors of voltage vector $V_{\text {out }}$ is also proposed. The key technology of realizing SVPWM algorithm will improve the dynamic response and steady-state accuracy performance of AC servo control system [4].

\section{PRINCIPLE AND REALIZATION OF SVPWM AL- GORITHM}

The ideal output of SVPWM is a three-phase symmetrical voltage source. The frequency and voltage can be

\footnotetext{
*Address correspondence to this author at the School of College of Electrical Engineering, Anhui Polytechnic University, Wuhu, Anhui, 241000, P.R. China; Tel: +86 553 2871262; Fax: +86 553 2871508; E-mail: 1by@ahpu.edu.cn,991by@sohu.com
}

adjusted. The SVPWM algorithm is used to reduce the harmonic component of the voltage source. A three-phase static coordinate system named $\mathrm{ABC}$ coordinate system, which was defined by motor's three-phase stator winding, has three shafts. The radian of these shafts is $120^{\circ}$. The stator phase voltages $U_{a}, U_{b}$ and $U_{c}$ were added on the motor's threephase stator winding and turned into three-phase voltage vectors. Directions of the three-phase voltage vectors are always stayed in its own axis. The sizes of the three-phase voltage vectors are changed according to the sine law. The three-phase voltage vectors are added up and turned into a voltage space vector named $U$. The rotation speed of the voltage space vector is an angular frequency of voltage source named $\omega[5,6]$. The calculation formula of $U$ is shown as follows.

$$
U=U_{a}+U_{b}+U_{c}
$$

In the $\mathrm{ABC}$ coordinate system, there is a one to one correspondence relationship between voltage vector and phase voltage. Therefore, there is a one to one correspondence relationship between coordinate and the nonzero voltage vector in the two-phase rotating coordinate system named $o \alpha \beta$ coordinate system. Sectors of the vector and the waveform of SVPWM are given in Fig. (1). Starting from $0^{0}$, which is the first sector, the value of sector is added to the last one by each $60^{\circ}$ interval according to the counterclockwise direction. The regulation of the sector is not the only one. The value of sector is defined as $0 \sim 5$ according to the counterclockwise direction in some documents. The order of tube angle of conduction and the best vector approximation group of SVPWM are given in each sector [7]. The calculation method of the time of the nonzero vectors needs to be considered as soon as the sector and the best vector approximation group are confirmed. In this study, the calculation method of seven-phase voltage space vectors $\frac{t 0}{4} \sim \frac{t 1}{2} \sim \frac{t 2}{2} \sim \frac{t 0}{2} \sim \frac{t 2}{2} \sim \frac{t 1}{2} \sim \frac{t 0}{4}$ is adopted. Therefore, the important work in this study is calculating $t 0, t 1, t 2$. 


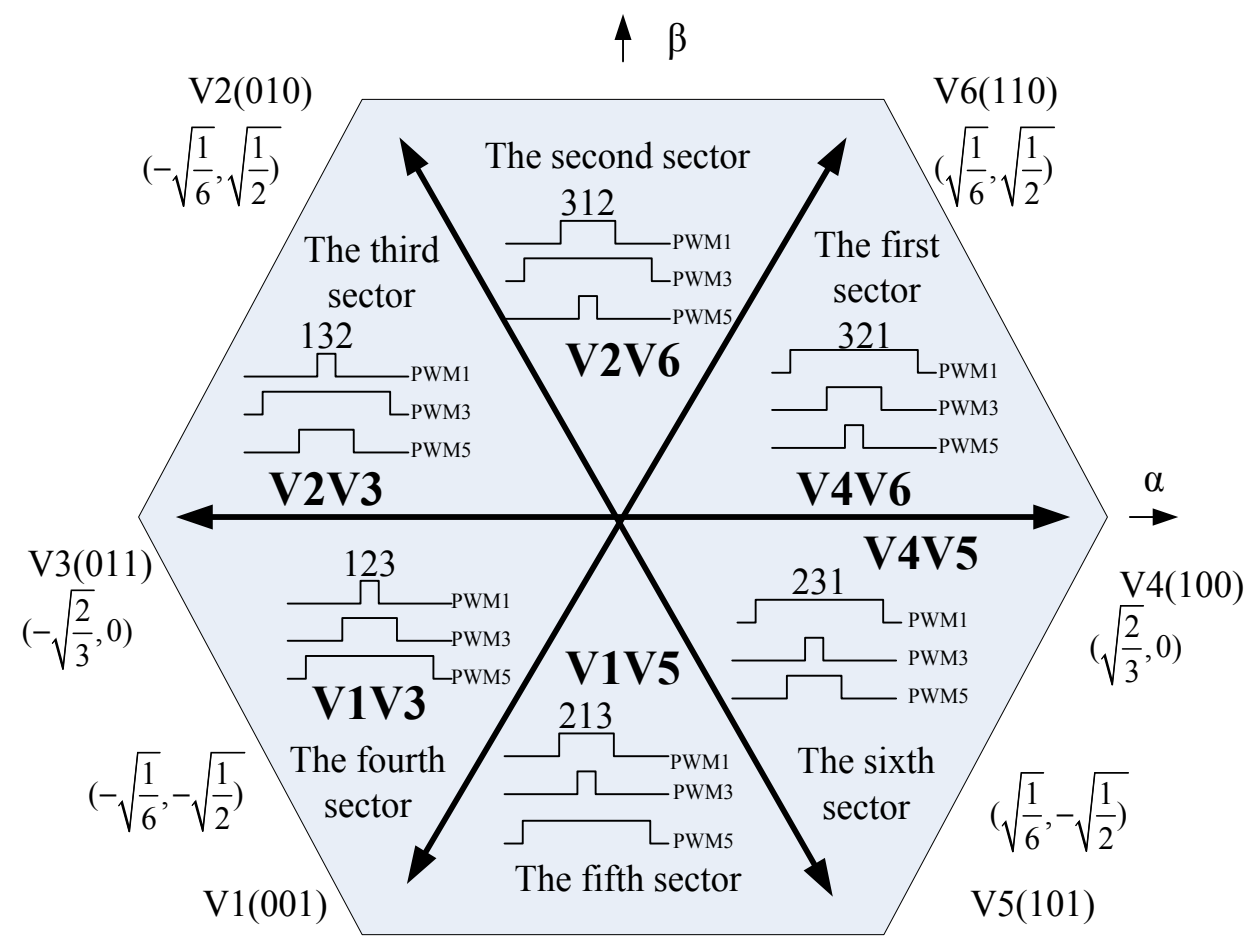

Fig. (1). Sectors of the vector and the waveform of SVPWM.

\section{CALCULATION METHOD OF NONZERO VEC- TOR MOVEMENT TIME $t 1, t 2$}

Calculation of the nonzero vector movement time $t 1, t 2$ is the key to the vector control algorithm. The following expression can be obtained when the projection of $U_{\text {out }}, U_{X}$ and $U_{x \pm 60}$ were projected onto the $O \alpha \beta$ coordinate system [8].

$$
\left[\begin{array}{l}
t 1 \\
t 2
\end{array}\right]=T_{P W M}\left[\begin{array}{cc}
U x \alpha & U_{x \pm 60 \alpha} \\
U x \beta & U_{x \pm 60 \beta}
\end{array}\right]^{-1}\left[\frac{V_{\alpha}}{V_{\beta}}\right]
$$

If the inverse matrix $\left[\begin{array}{cc}U x \alpha & U_{x \pm 60 \alpha} \\ U x \beta & U_{x \pm 60 \beta}\end{array}\right]^{-1}$ and the projection $\left[\frac{V_{\alpha}}{V_{\beta}}\right]$ of $U_{\text {out }}$ are known, we can calculate the nonzero vector movement time $t 1, t 2$. Therefore, we can obtain the calculation method of inverse matrix $\left[\begin{array}{cc}U x \alpha & U_{x \pm 60 \alpha} \\ U x \beta & U_{x \pm 60 \beta}\end{array}\right]^{-1}$ and projection $\left[\frac{V_{\alpha}}{V_{\beta}}\right]$ of $U_{o u t}$ by the following steps. The equation $\left[\begin{array}{ll}U x \alpha & U_{x \pm 60 \alpha} \\ U x \beta & U_{x \pm 60 \beta}\end{array}\right]\left[\frac{t 1}{t 2}\right] \frac{1}{T_{P W M}}=\left[\frac{V_{\alpha}}{V_{\beta}}\right]$ corresponds to the six sectors, and the values of $\left[\begin{array}{ll}U x \alpha & U_{x \pm 60 \alpha} \\ U x \beta & U_{x \pm 60 \beta}\end{array}\right]$ are given respectively as follows:

$$
\begin{aligned}
& {\left[\begin{array}{cc}
\sqrt{\frac{2}{3}} & \sqrt{\frac{1}{6}} \\
0 & \sqrt{\frac{1}{2}}
\end{array}\right],\left[\begin{array}{cc}
-\sqrt{\frac{1}{6}} & \sqrt{\frac{1}{6}} \\
\sqrt{\frac{1}{2}} & \sqrt{\frac{1}{2}}
\end{array}\right],} \\
& {\left[\begin{array}{lr}
-\sqrt{\frac{1}{6}} & -\sqrt{\frac{2}{3}} \\
-\sqrt{\frac{1}{2}} & 0
\end{array}\right],\left[\begin{array}{cc}
-\sqrt{\frac{1}{6}} & \sqrt{\frac{1}{6}} \\
-\sqrt{\frac{1}{2}} & -\sqrt{\frac{1}{2}}
\end{array}\right],\left[\begin{array}{cc}
\sqrt{\frac{2}{3}} & \sqrt{\frac{1}{6}} \\
0 & -\sqrt{\frac{1}{2}}
\end{array}\right] .}
\end{aligned}
$$

Therefore, we can calculate the inverse matrix $\left[\begin{array}{cc}U x \alpha & U_{x \pm 60 \alpha} \\ U x \beta & U_{x \pm 60 \beta}\end{array}\right]^{-1}$. By observing the product of the inverse matrix $\left[\begin{array}{cc}U x \alpha & U_{x \pm 60 \alpha} \\ U x \beta & U_{x \pm 60 \beta}\end{array}\right]^{-1}$ and the projection $\left[\frac{V_{\alpha}}{V_{\beta}}\right]$, twelve vectors are obtained. In these vectors, only six vectors are independent [9]. The calculation equations are given as follows: 
Table 1. Relational table between index number, sector and nonzero vector movement time.

\begin{tabular}{|c|c|c|c|c|c|c|}
\hline Index Number P & $\mathbf{1}$ & $\mathbf{2}$ & $\mathbf{3}$ & $\mathbf{4}$ & $\mathbf{5}$ & $\mathbf{6}$ \\
\hline \hline Sector N & 2 & 6 & 1 & 4 & 3 & $-\mathrm{X}$ \\
\hline $\mathrm{t} 1$ & $\mathrm{Z}$ & $\mathrm{Y}$ & $-\mathrm{Z}$ & $-\mathrm{X}$ & $-\mathrm{Y}$ & $-\mathrm{Z}$ \\
\hline $\mathrm{t} 2$ & $\mathrm{Y}$ & $-\mathrm{X}$ & $\mathrm{X}$ & $\mathrm{Z}$ & $-\mathrm{Y}$ \\
\hline
\end{tabular}

$\left[\begin{array}{ll}U_{0 \alpha} & U_{60 \alpha} \\ U_{0 \beta} & U_{60 \beta}\end{array}\right]^{-1}\left[\frac{V_{\alpha}}{V_{\beta}}\right]=\left[\begin{array}{ll}\sqrt{\frac{2}{3}} & \sqrt{\frac{1}{6}} \\ 0 & \sqrt{\frac{1}{2}}\end{array}\right]^{-1}\left[\frac{V_{\alpha}}{V_{\beta}}\right]=$ $\sqrt{2}\left[\begin{array}{cc}\frac{\sqrt{3}}{2} & -\frac{1}{2} \\ 0 & 1\end{array}\right]\left[\frac{V_{\alpha}}{V_{\beta}}\right] \begin{aligned} & -Z \\ & X\end{aligned}$

$\left[\begin{array}{ll}U_{120 \alpha} & U_{60 \alpha} \\ U_{120 \beta} & U_{60 \beta}\end{array}\right]^{-1}\left[\frac{V_{\alpha}}{V_{\beta}}\right]=\left[\begin{array}{ll}-\sqrt{\frac{1}{6}} & \sqrt{\frac{1}{6}} \\ \sqrt{\frac{1}{2}} & \sqrt{\frac{1}{2}}\end{array}\right]^{-1}\left[\frac{V_{\alpha}}{V_{\beta}}\right]=$ $\sqrt{2}\left[\begin{array}{cc}-\frac{\sqrt{3}}{2} & \frac{1}{2} \\ \frac{\sqrt{3}}{2} & \frac{1}{2}\end{array}\right]\left[\begin{array}{l}\frac{V_{\alpha}}{V_{\beta}}\end{array}\right] Y$

$\left[\begin{array}{ll}U_{120 \alpha} & U_{180 \alpha} \\ U_{120 \beta} & U_{180 \beta}\end{array}\right]^{-1}\left[\frac{V_{\alpha}}{V_{\beta}}\right]=\left[\begin{array}{rr}-\sqrt{\frac{1}{6}} & -\sqrt{\frac{2}{3}} \\ \sqrt{\frac{1}{2}} & 0\end{array}\right]^{-1}\left[\frac{V_{\alpha}}{V_{\beta}}\right]=$ $\sqrt{2}\left[\begin{array}{ll}0 & 1 \\ -\frac{\sqrt{3}}{2} & -\frac{1}{2}\end{array}\right]\left[\begin{array}{l}\frac{V_{\alpha}}{V_{\beta}}\end{array}\right] \begin{aligned} & Z \\ & -Y\end{aligned}$

$\left[\begin{array}{cc}U_{240 \alpha} & U_{180 \alpha} \\ U_{240 \beta} & U_{180 \beta}\end{array}\right]^{-1}\left[\frac{V_{\alpha}}{V_{\beta}}\right]=\left[\begin{array}{cc}-\sqrt{\frac{1}{6}} & -\sqrt{\frac{2}{3}} \\ -\sqrt{\frac{1}{2}} & 0\end{array}\right]^{-1}\left[\frac{V_{\alpha}}{V_{\beta}}\right]=$ $\sqrt{2}\left[\begin{array}{ll}0 & -1 \\ -\frac{\sqrt{3}}{2} & \frac{1}{2}\end{array}\right]\left[\frac{V_{\alpha}}{V_{\beta}}\right]_{Z}^{-X}$

$\left[\begin{array}{ll}U_{240 \alpha} & U_{300 \alpha} \\ U_{240 \beta} & U_{300 \beta}\end{array}\right]^{-1}\left[\frac{V_{\alpha}}{V_{\beta}}\right]=\left[\begin{array}{cc}-\sqrt{\frac{1}{6}} & \sqrt{\frac{1}{6}} \\ -\sqrt{\frac{1}{2}} & -\sqrt{\frac{1}{2}}\end{array}\right]^{-1}\left[\begin{array}{l}\frac{V_{\alpha}}{V_{\beta}} \\ ]\end{array}=\right.$ $\sqrt{2}\left[\begin{array}{cc}-\frac{\sqrt{3}}{2} & -\frac{1}{2} \\ \frac{\sqrt{3}}{2} & -\frac{1}{2}\end{array}\right]\left[\frac{V_{\alpha}}{V_{\beta}}\right] \begin{aligned} & -Y \\ & -Z\end{aligned}$
$\left[\begin{array}{ll}U_{0 \alpha} & U_{300 \alpha} \\ U_{0 \beta} & U_{300 \beta}\end{array}\right]^{-1}\left[\frac{V_{\alpha}}{V_{\beta}}\right]=\left[\begin{array}{cc}\sqrt{\frac{2}{3}} & \sqrt{\frac{1}{6}} \\ 0 & -\sqrt{\frac{1}{2}}\end{array}\right]^{-1}\left[\frac{V_{\alpha}}{V_{\beta}}\right]=$

$\sqrt{2}\left[\begin{array}{rr}-\frac{\sqrt{3}}{2} & \frac{1}{2} \\ 0 & -1\end{array}\right]\left[\frac{V_{\alpha}}{V_{\beta}}\right] \begin{aligned} & Y \\ & -X\end{aligned}$

\section{CALCULATION METHOD OF SECTOR OF VOLTAGE VECTOR $V_{\text {out }}$}

We can calculate the sector of voltage vector $V_{\text {out }}$ by the following method [11]. Definitions are shown as follows: 
Table 2. Relational table between index number, sector and timer time.

\begin{tabular}{|c|c|c|c|c|c|c|}
\hline Index Number $P$ & 1 & 2 & 3 & 4 & 5 & 6 \\
\hline Sector N & 2 & 6 & 1 & 4 & 3 & 5 \\
\hline$T_{c m 1}$ & $T b$ & $T a$ & $T a$ & $T c$ & $T c$ & $T b$ \\
\hline$T_{c m 2}$ & $T a$ & $T c$ & $T b$ & $T b$ & $T a$ & $T c$ \\
\hline$T_{c m 3}$ & $T c$ & $T b$ & $T c$ & $T a$ & $T b$ & $\mathrm{Ta}$ \\
\hline
\end{tabular}

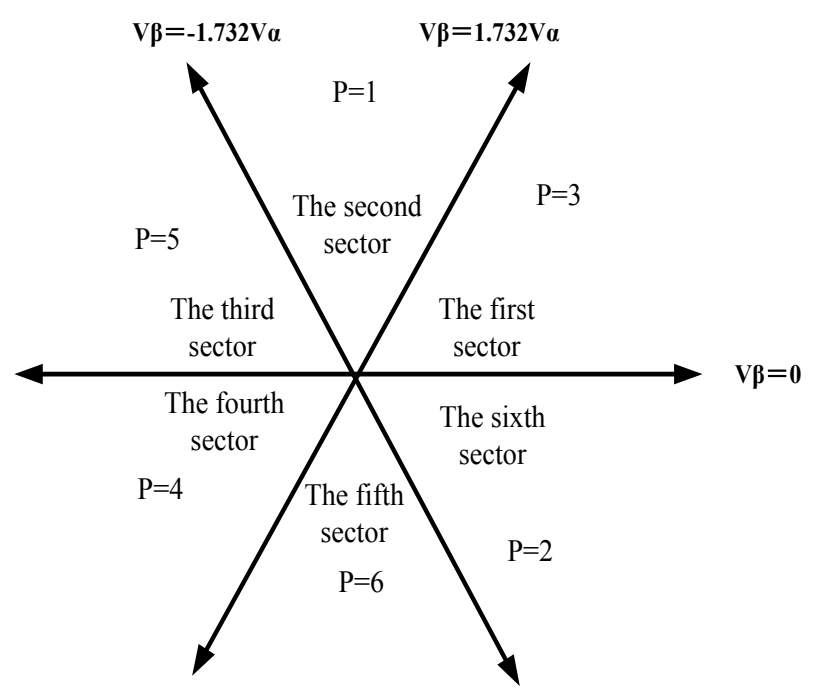

Fig. (2), Calculation diagram of sectors.

$$
\begin{aligned}
& B 0=V_{\beta} \\
& B 1=\frac{\sqrt{3}}{2} V_{\alpha}-\frac{1}{2} V_{\beta} \\
& B 2=-\frac{\sqrt{3}}{2} V_{\alpha}-\frac{1}{2} V_{\beta}
\end{aligned}
$$

Thus, we can calculate the index number $\mathrm{P}$ by the following formula:

$$
\mathrm{P}=4 \operatorname{Sign}(B 2)+2 \operatorname{Sign}(B 1)+\operatorname{Sign}(B 0)
$$

$V_{\alpha}, V_{\beta}$ are located in the rectangular coordinate system[12]. There are three lines in the rectangular coordinate system which are defined as follows:

$$
\begin{aligned}
& V_{\beta}=0 \\
& V_{\beta}=\sqrt{3} V_{\alpha} \\
& V_{\beta}=-\sqrt{3} V_{\alpha}
\end{aligned}
$$

Therefore, the calculation method for sectors can be obtained, as is shown in Fig. (2)

From Fig. (2), it can be seen that the $O \alpha \beta$ plane is divided into six regions, also known as sectors. Define that Sign $(B 0)=1$ in the plane above the line $V_{\beta}>=0$, on the contrary, Sign $(B 0)=0$. Define that $2 \operatorname{Sign}(B 1)=2$ in the plane under the line $V_{\beta}<=\sqrt{3} V_{\alpha}$, on the contrary, 2Sign ( $B 1$ ) $=0$. Define that $4 \operatorname{Sign}(B 2)=4$ in the plane under the line $V_{\beta}<=-\sqrt{3} V_{\alpha}$, on the contrary, 4Sign $(B 2)=0$. Therefore, we can calculate the index number by the values of $V_{\alpha}, V_{\beta}$ on the basis of the above definitions. Fig. (2) shows that there is a one-to-one relationship between the index number and the sector, so we can calculate the sector of voltage vector $V_{\text {out }}$ easily.

\section{SIMULATION}

Simulation was carried out on the system and motor parameters were selected as follows:

$$
\begin{aligned}
& R_{s}=4 \Omega, L s=34 \mathrm{mH}, \psi=0.51 \mathrm{~Wb}, \mathrm{~J}=0.05 \mathrm{~N} \cdot \mathrm{m}^{2}, \\
& R_{\Omega}=4 \mathrm{~N} \cdot \mathrm{s}, T_{j}=20 \mathrm{~N} \cdot \mathrm{m}, k_{f}=86, k_{g}=57 .
\end{aligned}
$$




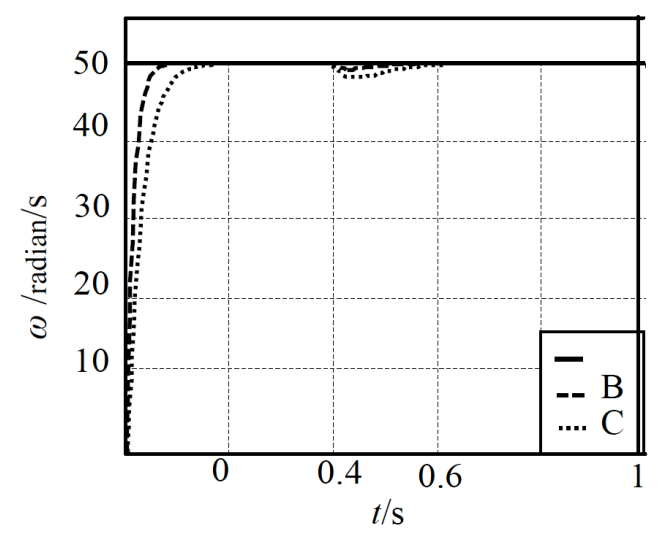

Fig. (3). Response curve of step signal.

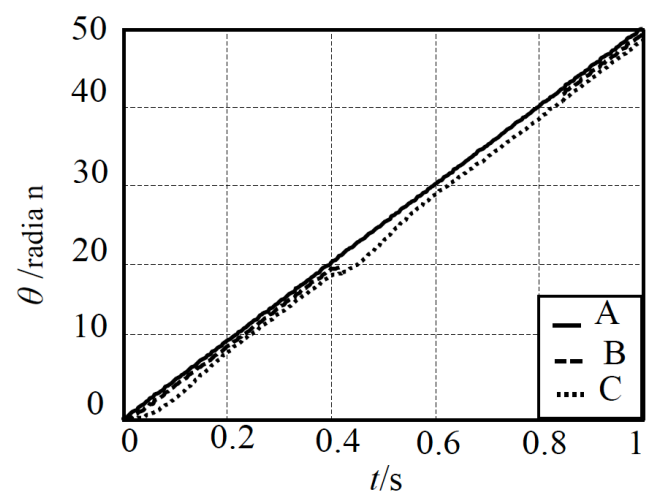

Fig. (4). Response curve of oblique wave signal.

We choose step signal and oblique wave signal as system input. The output of system is shown in Fig. (3) and Fig. (4). Curve $\mathrm{A}$ is the reference line and curve $\mathrm{C}$ is the response curve of conventional system. When adopting our proposed realization of SVPWM algorithm's key technology on the system, the reference line is shown as curve B. The simulation results demonstrate that the response time of curve $\mathrm{B}$ is about $0.054 \mathrm{~s}$ and the response time of curve $\mathrm{C}$ is about $0.063 \mathrm{~s}$. It also can be concluded that the recovery time of curve $\mathrm{B}$ is about $0.053 \mathrm{~s}$ and the recovery time of curve $\mathrm{C}$ is about $0.067 \mathrm{~s}$. Therefore, the anti-interference ability of curve $\mathrm{B}$ is better than curve $\mathrm{C}$.

\section{CONCLUSION}

In this study, a calculation method of sectors of the vector is given. Using projection source and matrix as tools, judging method of vector switch points $T_{c m 1}, T_{c m 2}, T_{c m 3}$ and calculation method of nonzero vector movement times $t 1, t 2$ is proposed. This study proposed a calculation method for sector of voltage vector $V_{\text {out }}$. Simulation was carried out and results show that the dynamic response time of system is improved by $16.7 \%$ and the precision of steady state response of system is improved by $20.7 \%$. The realization of SVPWM algorithm's key technology will provide a reference for future research. For this article, the future research work is to study the optimized algorithm and improve the control performances of system.

\section{CONFLICT OF INTEREST}

The author confirms that this article content has no conflict of interest.

\section{ACKNOWLEDGEMENTS}

This work was financially supported by the key project of natural science supported by education department of Anhui province (KJ2015A316), the major project of natural science supported by education department of Anhui province (KJ2014ZD004) and the outstanding young talents foundation project of Anhui Polytechnic University (2013RZR010).

\section{REFERENCES}

[1] H. Zang, Z. Qin, and Y. Dai, "Robust H-infinity Space Vector Model of Permanent Magnet Synchronous Motor Based on Genetic Algorithm," Journal of Computational Information Systems, vol.10, no. 14, pp.5897-5905, 2014.

[2] X. Jian, K. Zhang, and J. Chen, "An engineering design technique of PWM rectifier," Advanced Technology of Electrical Engineering and Energy, vol. 21, no. 3, pp. 44- 48, 2012.

[3] J. Wisniewski, and W. Koczara, "Poles position identification of the permanent magnet motor by the PIPCRM combined with zero voltage vector ," IEEE International Symposium on Industrial Electronics (ISIE), pp. 679-684, 2011.

[4] J. Yang, Y. Dai, and L. Yi, "DSP Based Equivalence of SVPWM and Carrier-Based PWM Study," Acta Electronica Sinica, vol. 38, no. 2010.

[5] L. Zhang, G. Zheng, and J. Li, "Dual-PWM Variable Frequency Control System of Mine Hoist," Journal of Computational Information Systems, vol. 9, no. 6, pp.2131-2137, 2013. 
[6] X. Qing, Z. Zhe, and Y. Xianggen, "A novel protection and control strategy for high-voltage power frequency control system," International Journal of Computer Applications in Technology, vol. 42, no.4, pp. 285-293, 2011.

[7] R. Yu, H. Zhang, G. Tan, and J. Zhang, "Study on H1Robust Control Method for PMSM," Power electronics, vol.46, no.2, pp. 102104, 2012.

[8] M. W. Qian, "Mixed H2/H1 control permanent magnet synchronous motor based on particle swarm optimization algorithm," Journal of Computer Applications, vol. 32, no.8, pp. 2381- 2384, 2012.

[9] J. Wang, K. Wu, Z. Liu, K. Wu, and Z. Zheng, "Research on High Power Factor Rectifier Applied to Electrical Vehicle Charger,"
Journal of Computational Information Systems, vol.9, no. 15, pp. 5947-5954, 2013.

[10] X. Jiao, and S. Li, "Adaptive speed tracking control of electric vehicles driven by permanent magnet synchronous motor," Electric machines and control, vol. 15, no.11, pp. 84-90, 2011.

[11] N. Hu, Y. Zhou, and J. Chen, "Study on Chaotic Control of SPWM Inverter and Its Optimization," Journal of Information and Computational Science Information Systems, vol. 9, no. 2. pp. 497- 504, 2012.

[12] L. Zhang, G. Zheng, and J. Li, "Direct Power Control Strategy of Three-phase PWM Rectifier," Journal of Information and Computational Science Information Systems, vol. 10, no.9, pp. $2807-$ 2813, 2013.

Received: July 01, 2015

Revised: August 23, 2015

Accepted: September 28, 2015

C Bingyou and Huicheng; Licensee Bentham Open.

This is an open access article licensed under the terms of the Creative Commons Attribution Non-Commercial License (http://creativecommons.org/licenses/by$\mathrm{nc} / 4.0 /$ ) which permits unrestricted, non-commercial use, distribution and reproduction in any medium, provided the work is properly cited. 